\title{
Intimate partner violence against women: the Peruvian case
}

Hong Kong Med J 2014;20:355

\section{DOI: $10.12809 / \mathrm{hkmj} 144335$}

To the Editor-In their original article, Ali et $\mathrm{al}^{1}$ state that in Karachi, the biggest city of Pakistan which is a country with an illiteracy rate of $45.1 \%,^{2}$ and one of the three countries with highest consumption of opiates, ${ }^{3}$ the factors most commonly associated with intimate partner violence (IPV) include illiteracy of women (odds ratio $[\mathrm{OR}]=5.9 ; 95 \%$ confidence interval [CI], 1.8-19.6), illiteracy of husbands $(\mathrm{OR}=3.9 ; 95 \% \mathrm{CI}, 1.4-10.7)$, current smoker status of husbands ( $\mathrm{OR}=3.3$; 95\% CI, 1.9-5.8), and substance use other than tobacco by husbands $(\mathrm{OR}=3.1 ; 95 \%$ CI, 1.7-5.7).

Peru is a South American country where more alcohol is consumed per capita $(8.1 \mathrm{~L})$ than the average consumption in the rest of the world $(6.1 \mathrm{~L}) .{ }^{4}$ According to Blitchtein-Winicki and ReyesSolari, ${ }^{5}$ alcohol consumption is the factor most commonly associated with IPV (OR=7.2; 95\% CI, 5.4-9.6), along with a history of physical aggression of the father towards the mother $(\mathrm{OR}=1.7 ; 95 \% \mathrm{CI}$, 1.4-1.9). Other factors associated with increased risk of IPV include having previous partners $(\mathrm{OR}=1.4$; 95\% CI, 1.1-1.7) and cohabiting $(\mathrm{OR}=1.4$; 95\% CI, 1.2-1.6). This apparent difference in factors associated with IPV could be explained by the ecological theory according to which, to properly approach this phenomenon, one must take into account the interaction of factors at different levels of analysis, namely, individual, family relationships, relationships with others, and social. Thus, in different cultures, we find the same phenomenon but different causes associated with IPV (Table). ${ }^{1,5}$

\section{Jhon W Mejía-Dolores *}

Sociedad Cientifica San Fernando, Universidad Nacional Mayor de San Marcos (UNMSM). Facultad de Medicina de San Fernando, Lima, Perú

* Corresponding author: jmejiad3@gmail.com

\section{References}

1. Ali NS, Ali FN, Khuwaja AK, Nanji K. Factors associated with intimate partner violence against women in a mega city of South-Asia: multi-centre cross-sectional study. Hong Kong Med J 2014;20:297-303.

2. UNESCO. Adult and youth literacy. National, regional and global trends, 1985-2015. Quebec: UNESCO Institute for Statistics; 2013. Available from: http://www.uis. unesco.org/Education/Documents/literacy-statisticstrends-1985-2015.pdf. Accessed Jun 2014.

3. UNODC. World Drug Report 2012. Viena: UNODC; 2012. Available from: http://www.unodc.org/documents/ data-and-analysis/WDR2012/WDR_2012_web_small.pdf. Accessed Jun 2014.

4. World Health Organization (2010b). Global status report
TABLE. Multivariate analysis of factors associated with intimate partner violence: results from two studies ${ }^{1,5}$

\begin{tabular}{|c|c|c|}
\hline Factors associated & P value & $\begin{array}{l}\text { Adjusted OR } \\
(95 \% \mathrm{Cl})\end{array}$ \\
\hline \multicolumn{3}{|l|}{ Pakistan ${ }^{1}$} \\
\hline \multicolumn{3}{|l|}{ Education status of participants } \\
\hline Graduate/postgraduate & & 1 \\
\hline Secondary/higher secondary & 0.25 & $1.9(0.6-5.8)$ \\
\hline Primary & $<0.001$ & $4.9(1.5-16.3)$ \\
\hline Illiterate & $<0.001$ & $5.9(1.8-19.6)$ \\
\hline \multicolumn{3}{|l|}{ Education status of husband } \\
\hline Graduate/postgraduate & & 1 \\
\hline Secondary/higher secondary & 0.37 & $1.5(0.6-3.5)$ \\
\hline Primary & 0.03 & $3.2(1.2-8.7)$ \\
\hline Illiterate & 0.01 & $3.9(1.4-10.7)$ \\
\hline \multicolumn{3}{|l|}{ Smoking status of husband } \\
\hline Never smoker & & 1 \\
\hline Ex-smoker & 0.01 & $3.0(1.3-7.1)$ \\
\hline Current smoker & $<0.001$ & $3.3(1.9-5.8)$ \\
\hline \multicolumn{3}{|l|}{ Substance use other than tobacco by husband } \\
\hline No & & 1 \\
\hline Yes & $<0.001$ & $3.1(1.7-5.7)$ \\
\hline \multicolumn{3}{|l|}{ Peru $^{5}$} \\
\hline \multicolumn{3}{|l|}{$\begin{array}{l}\text { History of physical aggression of the father towards } \\
\text { the mother }\end{array}$} \\
\hline No & & 1 \\
\hline Yes & $<0.001$ & $1.7(1.4-1.9)$ \\
\hline \multicolumn{3}{|l|}{ Alcohol consumption of partner } \\
\hline Partner does not drink alcohol & & 1 \\
\hline Partner drinks alcohol but does not get drunk & 0.56 & $0.9(0.6-1.3)$ \\
\hline Partner drinks alcohol and gets drunk sometimes & $<0.001$ & $1.8(1.5-2.2)$ \\
\hline Partner drinks alcohol and gets drunk continuously & $<0.001$ & $7.2(5.4-9.6)$ \\
\hline \multicolumn{3}{|l|}{ Has had previous partners } \\
\hline No & & 1 \\
\hline Yes & 0.008 & $1.4(1.1-1.7)$ \\
\hline \multicolumn{3}{|l|}{ Type of relationship } \\
\hline Married & & 1 \\
\hline Cohabiting & $<0.001$ & $1.4(1.2-1.6)$ \\
\hline
\end{tabular}

Abbreviations: $\mathrm{Cl}=$ confidence interval; $\mathrm{OR}=$ odds ratio

on alcohol and health 2014. WHO Library Cataloguing-inPublication Data. Luxembourg. Available from: http://apps. who.int/iris/bitstream/10665/112736/1/9789240692763_ eng.pdf. Accessed Jun 2014.

5. Blitchtein-Winicki D, Reyes-Solari E. Factors associated to recent intimate partner physical violence against women in Peru, 2004-2007 [in Spanish]. Rev Peru Med Exp Salud Publica 2012;29:35-43. 